Review

\title{
Engagement and Utilization of Advance Care Planning and Hospice in Different Older Asian American Populations
}

\author{
Kristy Lui $^{\dagger, *}$, Darlene Yee-Melichar ${ }^{\dagger}$, Emiko Takagi $^{\dagger}$
}

Gerontology Program, San Francisco State University, 1600 Holloway Ave, San Francisco, CA 94132, USA; E-Mails: kristylui@outlook.com; dyee@sfsu.edu; etakagi@sfsu.edu

$\dagger$ These authors contributed equally to this work.

* Correspondence: Kristy Lui; E-Mail: kristylui@outlook.com

Academic Editor: Lisa Hollis-Sawyer

Special Issue: Got Aging? Examining Later-life Development from a Positive Aging Perspective

\section{OBM Geriatrics}

2019 , volume 3 , issue 2

doi:10.21926/obm.geriatr.1902046
Received: October 31, 2018

Accepted: March 26, 2019

Published: April 08, 2019

\begin{abstract}
This research review sought to examine the patterns of engagement in advance care planning (ACP) and utilization of end-of-life (EoL) care treatment services including, but not limited to hospice care, in distinct older Asian American ethnic groups. The review of existing research suggested evidence that empirical studies about different older subpopulations of Asian Americans remains scarce. This research analysed 30 small-scale studies on older Asian Americans' attitudes and engagement in EoL care planning. The research review also synthesized the various obtained information, enabling better delineation of commonalities and differences between each unique subgroup. The review of selected articles indicated that the fear of becoming a burden to one's family and assumptions about EoL care attitudes between older adults and their family is a recurrent barrier to most Asian American subpopulations. However, the findings posit that variations exist amongst Asian American cultures despite some overlapping qualities. Thus, an urgent need for more research about $\mathrm{ACP}$ and EoL care treatment services in different older Asian American communities persists. Lastly, culturally and linguistically sensitive interventions are necessary to increase ACP
\end{abstract}

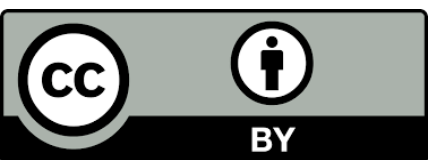

(C) 2019 by the author. This is an open access article distributed under the conditions of the Creative Commons by Attribution License, which permits unrestricted use, distribution, and reproduction in any medium or format, provided the original work is correctly cited. 
participation in different older Asian American populations as a way to improve their quality of life.

\section{Keywords}

Asian Americans; advance care planning; advance directive; death; dying; end-of-life; hospice; late life

\section{Introduction}

The human population is rapidly aging and living longer than ever before, raising a need for higher quality geriatric care and increased participation in advance care planning (ACP). ACP is a process involving active communication that enables a person to consider what kind of care they would like to receive and learn what treatments can assist them with achieving their desired care. The goal of ACP is to ensure that people receive the kind of care that is consistent with what quality of life means to them, rather than focusing on the quantity of time. This mission is accomplished by understanding the individual's unique values, personal goals and preferences for care, and then carrying them out in circumstances of incapacitation [1]. Moreover, ACP is an important facilitator for communication between the individual and their family, loved ones, and healthcare practitioners in order to understand and share their "personal values, life goals, and preferences regarding future medical care" [1]. While ACP is more often than not associated with older adults and those with life-threatening illnesses nearing the EoL, the process of discussing and planning one's care is available and encouraged for people of all ages and stages of health [1].

Granted, ACP discussions that focuses on care at the EoL are especially important to the older population, which is projected to grow dramatically in numbers and become increasingly more racially and ethnically diverse [2]. As people are living longer, older individuals are also more likely to have, or to develop at least one chronic illness in later age that results in their death [3]. In fact, chronic illnesses are now the leading causes of death in the U.S. population, including heart disease and cancer [4]. However, chronic diseases are for the most part manageable with the appropriate medical care and general healthy lifestyle changes, which can enable individuals to live extended lives. Therefore, older adults today should have a better advantage and more time to plan their EoL care even while simultaneously managing chronic illnesses compared to the past. However, research studies have indicated that the general population and especially older adults are not actively engaging in EoL discussions or planning [5]. For the purpose of this research, the discussion of ACP focuses on care at the EoL in the growing geriatric population.

\subsection{Benefits of Advance Care Planning and Advance Directives}

The benefits and importance of ACP have been consistently emphasized by dying patients, caregivers and healthcare advocates as an integral key to achieving the quality of life and death that follows an individual's preferences and respects their autonomy [3]. ACP not only fosters respect for the individual's autonomy, but it also prepares individuals for possible scenarios of future incapacity, and facilitates the completion of a formal advance directive (AD) - a legal 
document providing direction for healthcare decisions [6, 7]. According to Detering et al. [6], "patients see the benefits of advance care planning to include preparation for end of life care and death, avoidance of prolongation of dying, strengthening personal relationships, relieving burdens placed on family, and the informal communication of future wishes" (p. 5). While this may be true, existing research generally suggests that significantly low participation in ACP and utilization of ADs or other formal documents continue to persist [8].

Early involvement in EoL conversations and ACP is crucial because the probability of incapacitation can be unpredictable but possible. According to Carr and Luth [3], about 45\%-70\% of older adults facing the EoL are unable to make treatment decisions for themselves and thus, family members and other loved ones assume the responsibility instead. Furthermore, the lack of prior decision-making by the patient and the likelihood of family members being in a state of dejection may contribute to disagreements amongst each other about what treatment, if any, is suitable for their dying loved one. When an agreement cannot be reached between the family and the health care providers, the continuation of life-sustaining treatment is carried out which can pose emotional and financial hardship to families and jeopardize their loved one's quality of life [3]. By engaging in early EoL conversations and ACP with family, healthcare providers, and legal professionals, situations like these are more likely to be avoided.

\subsection{Attitudes towards Death and Dying}

Despite the benefits of $A C P$, more than half of the American population lack advance care plans and the low prevalence of EoL conversations between patients and health care providers persist [9, 10]. Present findings reveal that a considerable number of Americans would prefer to die at home and not burden loved ones with EoL care decision-making [11]. However, in a study examining the usage of $A D$ in the U.S., only $26.3 \%$ of 7,946 respondents reported having completed one [8]. According to Hamel, Wu, and Brodie's (2017) cross-country examination of different cultural attitudes towards EoL planning, only eleven percent of Americans reported ever having such a conversation with a physician or health care provider. Furthermore, only twenty-two percent of those Americans 65 and older reported having those EoL conversations [12]. These results have often been explained to be a product of today's death-phobic society.

Correspondingly, death and dying is generally looked upon unfavourably and is treated as a topic to be avoided at all cost. According to Hamel et al. [12], more than half of Americans believe death is a subject that is mainly avoided in the U.S. society. Above all, the phenomenon of death and dying have evolved from a community experience to an institutional-based event over timecontributing to today's death-phobic culture and society [13]. The event of death and the process of caring for the dying have transformed from a strictly familial matter to one that is executed by professionals and where death now occurs primarily in hospital settings instead of one's own home [13]. Additionally, the displacement of cemeteries to areas away from the central population, new life-prolonging technology, and generally longer life expectancies have allowed individuals to momentarily set aside the thought of death and dying until further in the future.

Likewise, an individual's general apprehension towards the EoL often presents a sense of disinclination and a personal barrier from participating in ACP. Fear of facing one's morality, concerns of burdening family members, and a general disbelief that one is capable of planning for the unpredictable future are some common reasons that patients given for not partaking in ACP 
[10]. As a result, fewer conversations about the EoL occur in both public and private settings, generating poorer communication and understanding of care and treatment preferences during this time.

Additionally, other major contributors hindering accessibility to ACP and the general process is in part due to a lack of knowledge about EoL care services, an absence of culturally appropriate EoL care resources, and poor communication about the EoL between older adults, their family, and health care professionals [14-17]. Thus, it is to be expected that the general population's participation in ACP remain at a constant low. Moreover, it is anticipated that members of ethnic minority groups residing in the U.S. exhibit even lower rates of engagement in ACP or engage in EoL care services, such as hospice, due to differing cultural beliefs and other inhibiting factors. The lack of engagement in ACP and EoL care services is particularly salient amongst minority populations such as older Asian Americans.

\subsection{Older Asian Americans in the United States}

Asian Americans are one of the ethnic minority populations whose pattern of engagement in EoL care planning remains substantially understudied despite their growing numbers. According to López, Ruiz, and Patten [18], the Asian American population living in the United States increased from 11.9 million to 20.4 million between the year 2000 and 2015. Moreover, Asian Americans alone made up 5.8 percent of the total American population of 325 million in 2017-deeming them the fourth largest ethnic group [19]. The Asian American population is composed of 19 different origin groups, each with distinct cultural values and practices [20]. The largest subgroups include: Chinese Americans, Filipino Americans, Japanese Americans, Korean Americans, and Vietnamese Americans being the largest and most prominent communities.

Furthermore, there are some common characteristics among the older Asian American population. Asian Americans have a generally longer life expectancy than other ethnic populations at about 86.3 years, outliving their non-Asian counterparts of about eight years [21]. Many of these individuals are immigrants, lack English fluency, and live below the poverty level [22]. Studies have also indicated that cancer, heart disease, diabetes and suicide are the top leading causes of death for Asian Americans [22]. However, it is important to remember that Asian Americans "are a heterogeneous population that includes diverse cultural backgrounds and immigration histories," as well varying socioeconomic statuses, that have in turn shaped their health and access to health care [22]. Hence, it would be inappropriate to generalize the health and health care issues faced by each subgroup to the entire Asian American population.

Correspondingly, previous literature shows that members of minority communities tend to have a significantly lower rate of participation in ACP and hospice care [23]. As reported by NgoMetzger et al. [23], older foreign-born Asian Americans were less likely to use hospice compared to U.S. born Caucasians. In 2015, of 48 million aged Medicare recipients, 81.4 percent were Caucasian, 10.1 percent were African American, 9.3\% were Hispanic or Latino, and 2.2\% were Asian [24]. In a report by the National Hospice and Palliative Care Organization (NHPCO), it was found that of 1,381,182 Medicare beneficiaries who were enrolled in hospice services in 2016, about 87 percent were Caucasian compared to 8.2 percent African American, 2 percent Hispanic, 1.2 percent Asian American, and 0.4 percent Native American [25]. This significant discrepancy in participation among ethnic groups indicate that there is a dire need for further investigation. 
Moreover, with Asian Americans being one of the fastest growing and aging minority population in the United States, we can expect an increased demand for providing culturally appropriate support to these aging individuals as they reach their final moments of life-making it even more crucial to examine the attitudes and patterns of engagement in EoL care within the different subpopulations.

\section{Materials and Methods}

\subsection{Research Purpose}

The purpose of this research review was to examine the patterns of engagement in ACP and the utilization of EoL care treatment services in distinct older Asian American ethnic groups. The importance of this research stems from several observed concerns. The EoL is an area that continues to receive insufficient attention in both Eastern and Western cultures. However, the attitudes and preferences towards ACP and hospice utilization in older Asian American subgroups have been especially inadequately explored with only a handful of research specifically examining these areas $[17,26]$. Moreover, data pertaining to ACP participation and hospice use by older Asian Americans is limited thus far and existing research tends to overgeneralize and classify the experiences of these diverse subgroups into one broad category-despite the heterogeneity of each community. The overgeneralization of the EoL care research contributes to the lack of clarity about the diverse attitudes and preferences that each Asian American subpopulation holds towards ACP and its supporting services. Presently, extant data generalizing these communities cannot be representative of all Asian American subgroups.

Moreover, there is a need to synthesize the current available information and findings from small-scale studies about Asian American ethnic groups and EoL care to delineate commonalities and differences between the subpopulations. By having a clearer overlook of the present findings, we can gain a more coherent understanding of the status of EoL care planning participation in older Asian American subgroups. This will in turn support the facilitation of further research and advances to change patterns of engagement in these ethnic groups. In the following research review, the utilization and engagement in ACP and hospice care by older Asian American subgroups are examined to gain a more defined understanding of the unique attitudes, preferences, barriers and cultural factors that influence decision-making at the EoL.

\subsection{Research Methods}

A review was conducted to examine the existing research on older Asian American subpopulations' attitudes, preferences and participation in ACP and hospice care. Past research studies and reports were first collected by accessing various databases and search engines, including Ebsco, Google Scholar, JSTOR, NCBI, PubMed, SAGE Journals Online, ScienceDirect, SpringerLink, and Wiley Online Library. Keywords including 'Asian Americans' and their subgroups, 'advance care planning', 'death', 'dying', 'end of life', 'elderly', 'hospice', 'late life', and 'older age' were then entered into the databases to obtain the relevant studies. The abstracts of each result rendered by the databases were examined to determine the relevance of the content to the research subject, as well as its overall quality, before moving on to the next stage of the reviewal process. Articles that did not include or discuss one or more of the key terms mentioned above 
were ultimately excluded due to lack of relevance to the topic of this review. Moving forward, articles that passed this initial review were then explored in greater depth of their content quality to decide whether their findings adequately and sufficiently addressed Asian American subpopulations and their engagement in ACP and EoL care treatment, as well as their cultural attitudes and preferences regarding death and dying.

Review articles included any previous research examining subpopulations of Asian Americans and ACP, AD, hospice care, and other EoL care treatments. Articles studying these communities' attitudes towards death and dying, as well as pain-management at the EoL were also included. Moreover, due to the limited amount of research available for each subpopulation, the time of publication for each article was not limited. Additionally, the collected data was not limited to those who are or were diagnosed as terminally ill. After filtering all potential studies through the outlined criteria, a total of 30 articles were selected for review.

\subsection{Definition of Concepts}

\subsubsection{Advance Directives (AD)}

An $A D$ is a legal document that allows individuals to provide family members, loved ones, medical professionals and legal figures with instructions on how they would like to be treated at the EoL in case they were to lose the ability to personally deliver them at the time [7, 27]. For instance, the decision to utilize or forego life-sustaining treatment at the EoL is one of the available options in an $A D$ that individuals can choose to opt in or out of. In addition, ADs also enable individuals to designate another person to make decisions on their behalf-also known as a surrogate, proxy directive or durable power of attorney for health care [7]. Individuals can also continuously change and update their ADs as they wish and see fit.

\subsubsection{End-of-life Care Treatment and Services}

The various treatments that an individual may receive at the EoL is also known as life-sustaining or life-withholding treatment. According to Idziak (2002) [28], life-sustaining or life-withholding treatment can be any medical intervention or medication that is administered to a patient to delay the event of death, regardless of whether or not the treatment is intended to influence the disease itself or the natural biological process of dying. For this discussion, EoL care encompasses external treatments including chemotherapy, kidney dialysis, resuscitation, ventilator use, and artificial tube feeding, as well as more internal treatments, such as the administration of antibiotics or other pain management medications [28]. Palliative care and hospice care are broader programs that may also include all the above treatments, and thus, will fall under the EoL care services category in this review.

\subsubsection{Hospice and Palliative Care}

Correspondingly, the goals of hospice are aligned with those of ACP in which both strive to provide care that is consistent with the individual's desires. Hospice is a form of all-inclusive care offered at the EoL that focuses on improving one's quality of life through providing comfort-based, rather than cure-oriented treatment, and by following the individual's unique preferences. As part 
of the push for more comfort-based care at the EoL, Medicare beneficiaries are eligible to receive hospice care at a covered cost. In 2014, 85.5 percent of hospice patients in the U.S. were covered by Medicare [29]. In addition, hospice brings together a multidisciplinary team of professionals who each provides a variety of medical, psychological, spiritual, and bereavement support to the individual and their family. Eligible individuals can opt to participate in hospice care when engaging in ACP. In fact, hospice care can be provided in a variety of settings, including "free standing hospice facilities, hospitals, and nursing homes and other long-term care facilities" [25]. Individuals who enroll in this care service tend to have a terminal illness and is diagnosed with an estimated six months left to live by a medical professional. Palliative care, or pain management, is often associated with hospice care where the distinct difference lies in the diagnosis; an individual need not to be terminally ill to be able to utilize palliative care. Additionally, palliative care differs from hospice where pain management is simultaneously compatible with curative treatments.

\section{Results}

Of the 30 studies that matched the inclusion criteria, 27 articles and their findings were ultimately selected for this scoping review. The qualitative study designs and methodologies reported in the reviewed articles included the use of self-administered questionnaires and surveys, as well as individual interviews and focus groups, to either recruited participants or convenience samples. Subsequently, the participants in the collected samples primarily focused on older Asian Americans who resided in or passed away in the states and are aged 65 years or older. Younger individuals were also examined in these studies as a comparison to the older generations of Asian Americans. Research studies analysing a general Asian subculture, as well as older Asians living overseas were also examined, as there were overlapping beliefs and practices that these individuals and older Asian Americans shared.

The findings from each selected article were then organized by the researchers into four primary categories representing the overlapping themes identified during the reviewing process: (a) attitudes towards ACP and EoL support services, (b) influential cultural practices, including religious and spiritual beliefs, (c) preferences for EOL treatment, and (d) perceived barriers to accessing and engaging in these practices. The researchers then entered the results into a structured table highlighting the primary findings in each category within the subpopulations (see Table 1). The gathered data was critically analysed to determine the dominant and recurrent trends between older Asian American ethnic groups. Both similarities and discrepancies are discussed in the following sections.

Although the amount of research studies on older Asian Americans' attitudes and participation in ACP have grown over time, the existing research still insufficiently addresses all the different subpopulations as distinct communities with unique preferences and needs. The collected data was heavily concentrated on older Chinese, Japanese, and Korean Americans due to a large absence of available information on the other subgroups. Research about older Chinese Americans and ACP consistently appeared when using the different databases despite specifically searching for other distinct subpopulations. Furthermore, studies addressing older Filipino and Vietnamese American communities regarding EoL care were extremely limited compared to the other three subgroups. 


\subsection{Attitudes towards Advance Care Planning and End-of-Life Care Services}

To begin, the existing research studies have found varying attitudes towards ACP and EoL care services amongst the different older Asian American subpopulations. In a 2017 study comparing younger and older Chinese Americans' beliefs in ACP, it was discovered that the elderly individuals were more accepting of ACP compared to the younger participants and also believed that it was necessary regardless of one's state of health [17]. A number of elderly participants also had the impression that the desired outcome of ACP is to lighten the burden of EoL decision-making on their loved ones and medical professionals [17]. However, studies have also indicated a recurring notion of misconceptions by elderly Chinese Americans regarding what ACP and EoL care services entail. For example, ACP and hospice care is often perceived to be a form of euthanasia by members of this older subpopulation [17, 30]. According to a study by Enguidanos et al. [30], older Chinese adults perceived hospice care as "mercy killing," comparable to assisted suicide or euthanasia. Lee et al. [17] had similar findings in which the older Chinese participants confused ACP with euthanasia, creating a will, and/or making funeral arrangements. These misconceptions are not uncommon amongst ethnic minority communities, like Asian Americans, where access to culturally and linguistically-appropriate resources may be largely unavailable [15, 31].

Correspondingly, research on older Korean Americans' attitudes towards ACP and EoL care also demonstrated an overall lack of awareness and misinterpretation of what the services involve. As reported in the 2015 Asian American Quality of Life Survey, only 5.6 percent of Korean Americans had completed an AD [32]. Previous investigation have suggested that older Korean Americans are often considered a homogenous group where the majority were born, raised, and reached older ages in Korea before immigrating to the United States [33]. Therefore, it can be implied that members of this community are more likely to share common cultural values, adhere to their own native culture, and hold identical beliefs to older Koreans living overseas [33]. Although existing studies on older Korean Americans and $A D$ is limited, the findings from these studies have consistently demonstrated low completion rates of ADs and that members of this community are unaware or hold misunderstandings of its intentions [33]. In a study conducted in 1996 examining ethnic variations in $A D$ completion, zero percent of older Korean adults residing in the Los Angeles County had completed an $A D$, compared to five percent in more recent findings [33, 34]. According to Park [35] who compared ACP among Korean and Korean American focus groups, he found that many older Korean and Korean Americans, as well as their family and/or caregivers, expected that life-sustaining treatment--an option provided in $A D$, would cure one's condition or prolong the patient's life. Much like other Asian American subgroups, many older Korean adults are immigrants and lack English fluency, thus impacting their understanding of health and health care services like those provided at the EoL. Therefore, hesitancy to participate in ACP and opting for, or out of certain EoL care treatments can be anticipated from this ethnic group due to insufficient knowledge of available health care services.

Similarly, although research on older Filipino American adults and their perception of the EoL is limited, the existing findings have consistently indicated that members of this subpopulation overall may hold more positive attitudes towards ACP compared to other Asian Americans [36]. However, few older Filipinos have engaged in ACP or the discussion of EoL treatment and often feel uncomfortable planning for death [37]. Despite the general lack of participation in ACP and EoL discussions, older Filipino Americans were found to have a significantly higher completion rate 
of $A D(22.4 \%)$ compared to Chinese (14\%) and Korean Americans (5.6\%) [32]. According to the 2015 Asian American Quality of Life Survey project by Jang [38], Filipino American participants were also found to have the highest rate of awareness about $A D$ and its related services compared to other Asian American subgroups.

Correspondingly, EoL discussions are also generally frowned upon in the Vietnamese culture, much like their Asian American counterparts [39]. In a Stanford report examining Vietnamese participants residing in Hawaii and EoL care, it is common practice among older Vietnamese Americans to make solid preparations for their death although general discourse about death and dying is viewed negatively and seen as an unfortunate event [39]. These arrangements include "setting aside money to pay for the burial, choosing a burial site with a favourable orientation in accordance with the laws of feng shui, buying a coffin, and even buying or having burial clothes made long before they are actually needed" [39]. Moreover, the act of preparing for one's own death is recognized as the older adult's responsibility to organize for themselves, instead of their children [39]. However, the concept of ACP and formal documents such as AD are still foreign and unfamiliar services that most Vietnamese families are unaware of [39].

Contrastingly, Japanese American older adults have been found to hold more positive perceptions of ACP and have generally higher awareness of $A D$ and other EoL care services compared to other Asian Americans [40]. The research study indicated that third generation Japanese Americans (sansei) are more open to having EoL care conversations in contrast to the first (issei) and second (nisei) generations [40]. These findings may be due to the differing levels of acculturation with each successive generation holding more Westernized values and higher English proficiency. In a study conducted by Matsumura et al. [40], 11 percent of English-speaking Japanese Americans (EJA) ( $n=539)$, nine percent of Japanese-speaking Japanese Americans (JJA) $(n=340)$, and six percent of Japanese-speaking Japanese $(\mathrm{JJ})(\mathrm{n}=304)$ had ever discussed their EoL care with a physician [40]. Furthermore, it was also found that 40 percent of EJA, 55 percent of JJA, and 54 percent of JJ expressed the desire to have these discussions. However, 18 percent of EJA, 16 percent of JJA, and 23 percent of JJ said they did not wish to discuss their EoL care with their physician [40]. Overall, EJA were found to be more favourable towards foregoing treatment and planning their EoL care [40]. The study's findings demonstrated a strong correlation between acculturation, engagement in ACP and preferences for EoL treatment that may be applicable to ethnic minority communities. Other studies looking at different Asian American populations have also found similar results in ACP and EoL care preferences, including older Korean immigrants [35].

\subsection{Cultural Beliefs and Practices}

Based on the collected research, several cultural beliefs, practices and degree of acculturation were identified as playing an important role in the shaping of older Asian American adults' attitudes and preferences towards ACP and EoL care. The research demonstrated similarities with slight variations between the different Asian American groups including: cultural superstitions, religious and spiritual beliefs, collectivism and the importance of filial piety.

\subsubsection{Cultural Superstitions}

In accordance with the current research, mixed attitudes by older Asian Americans towards death, dying, and pain were recognized as affecting ACP and EoL care engagement. The 
foundation for these attitudes were discovered to be a combination of cultural superstitions, as well as spiritual and religious beliefs. For example, death and illness are treated as serious taboo that would bring about misfortune, such as casting death upon the individual and/or their family by older Chinese and Filipino adults [37, 41]. Within the Chinese culture, "death is considered mysterious and frightening; it is considered impolite and bad luck to discuss" [37]. Furthermore, some research have demonstrated that the Filipino culture believes discussing death-related matters or simply speaking about death itself may prompt unwanted consequences and fear upon the elder and their family [36]. Similarly, discussion of death is also deemed disrespectful and even traumatizing towards a dying patient in Korean culture [35]. Therefore, the negative cultural beliefs towards death may in part influence older Asian American ethnic groups' attitudes and preferences for EoL care.

\subsubsection{Religion and Spirituality}

Moreover, existing research indicates that older Asian Americans who value religion and spirituality hold differing views of pain and death than those who do not. Notably, members of both Filipino and Vietnamese culture were found to perceive illness and death to be inevitable and as a will of God or a higher power $[16,36]$. The popular Filipino term bahala na illustrates the idea that life events, such as death and pain when dying, are destined or inevitable [36]. Similarly, pain is traditionally believed to be a natural product of dying and individuals are expected to display a sense of patience while experiencing pain [16]. Identically, death is also perceived as a natural event in the life cycle in Japanese and Vietnamese culture [39, 42]. As reported by Duke and Petersen [16], pain is to be endured as it is a "cultural value and spiritual expectation...to remain virtuous in the moment of death" and serves as a method to atone for one's sins in the Vietnamese culture (p. 31). According to Tran and Hinton [39], the perception of death and pain by older Vietnamese immigrants in the U.S. may largely be the result of Catholicism or Buddhism, but also the practice of Confucianism, Animism, and Taoism by members of this community [39, 43, 44]. As stated by Tran and Hinton [39], these religious and cultural/societal norms influence how death is perceived and enables individuals to re-examine the event in a more positive light. For example, "concepts of reincarnation are prevalent among Buddhists, ancestor worship, and the belief of going to heaven after death, may cause death to be viewed as something fortunate, and fitting with life's natural cycle" [39]. Additionally, according to Shin et al. [45], older Koreans who practice Confucianism may more likely prefer to discontinue life-prolonging treatment to experience a natural death that is consistent with their religious practices. Similar experiences of comfort with EoL care discussions were found in older Japanese Americans as the Buddhist ideology reinforces the conversations about death [37]. Therefore, it is suggested that where cultural and religious beliefs are embodied, the attitudes towards death and ACP may be significantly impacted. Ethnic individuals who believe death is an inevitable or a natural occurrence in life may be more likely to forego life-sustaining treatment and be more open to participating in ACP compared to those who do not hold such beliefs.

\subsubsection{Collectivism and Filial Piety}

The different ethnic Asian cultures are largely collectivistic in which one's relationship to other individuals is valued and prioritized instead of the individual themselves when making important 
life decisions [37]. Collectivism is one of the many attributes that characterizes the Eastern culture and sets it apart from the individual-oriented Western society. Whereas the Western culture often focuses on the promotion of the individual's well-being and cultivating independence, maintaining intergroup harmony within the family and society are the fundamental features of the Eastern culture. Asian culture is widely described as collectivistic-oriented where members of this community tend to be more concerned about others' feelings and thoughts [37]. Chinese, Filipino, Japanese, Korean, and Vietnamese cultures have commonly been noted as primarily collectivistic $[17,33,44,46,47]$.

According to Chew [37], the importance of family and the in-group encompass Asian Americans' sense of self. At the EoL, Asian Americans "tend to be more concerned about whether or not their family and significant others will be overly burdened, happy, or taken care of during the crisis period that precedes their death and the transition period after death" [37]. The worries of these individuals are often believed to derive from the traditional beliefs and practices of filial piety in Asian American culture.

Moving forward, filial piety is an overarching notion stemming from Confucian teachings that significantly impacts the attitudes, decision-making and preferences for EoL care for many Asian American families. The concept of filial piety is generally understood to be a cultural obligation of family members to care for and respect one another [36]. This cultural responsibility posits that adult children are obligated to eternally care for their parents and protect them from any possible harm [48]. Filial piety encompasses the overall definition of collectivism where interrelationships are prioritized over the self. For example, one's relation to family and society has a significant role in how or what kind of EoL care older Filipino Americans may choose for themselves. Oftentimes, to avoid conflict or disagreement among family and the community, Filipinos may go along with the decision made for them even if they do not agree with it [36]. Older Korean Americans also share a cultural expectation of filial piety (hyodo in Korean), where it is assumed that adult children are expected to protect and carry out decisions involving their parents' physical and emotional well-being [33, 49]. Blackhall et al. [49] found that older Korean Americans "did not believe their personal desires for life support were relevant to treatment decisions" (p. 1787).

Moreover, in accordance with traditional family hierarchy, there is a large emphasis on the eldest son as the most valued child and generally younger individuals are expected to respect parents and elders who are traditionally assumed to possess abundant life experience and knowledge and therefore should be revered [36]. The current findings demonstrate that filial obligation significantly plays a role in the decision-maker and the process of making EoL choices in all the older Asian American subgroups [17, 36, 42, 44, 49].

To reiterate, the discussion of death with the dying older adult is considered disrespectful and culturally unacceptable in most Asian American subgroups because it ultimately defies the cultural expectations of filial obligation and disrupts intergroup harmony [17, 35, 37]. This in turn creates conflict and confusion between the older adult and their children. While older Asian Americans generally expect their adult children to make EoL care decisions for them, the cultural taboo hinders the necessary communication from both parties. The current findings indicate that the traditional concept of filial piety remains an important practice where older Asian Americans expect family members, particularly children, to be the ultimate decision-maker regardless of their degree of acculturation. For instance, when one is ill in the Filipino culture, a passive role is assumed and individuals are expected to receive family support [36]. Traditionally, the eldest son 
in both Filipino and Vietnamese are usually consulted first by physicians and are expected to carry out most of the decision-making involving the parents' care [36, 39]. The current research indicates that filial piety remains a pivotal component influencing older Asian Americans' involvement in ACP and EoL care discussions.

However, family members tend to fulfil their sense of filial obligation without fully understanding their loved one's wishes at the EoL. As noted earlier, the discussion of death with the dying older adult is considered disrespectful and unacceptable in a majority of the examined Asian American subgroups [17, 35, 37]. This in turn creates conflict and confusion between the older adult and their children. While older Asian Americans generally expect their adult children to make Eol care decisions for them, the taboo of discussing death hinders the necessary communication from both parties. Park [35] found in their study on older Korean Americans that adult children are expected to already know their wishes even without explicit discussions about Eol care. Additionally, the family members reported that they understood their parent's preferences through inferences gathered from parents' informal comments about the EoL when they came up from time to time [35]. Instead, it is common practice to not engage in "truth telling" with their dying family member in the case of impending or incurable illness to avoid unnecessary grief or discomfort [37]. Research on older Chinese and Korean Americans and their families indicated that the act of consulting with the elderly individual about the EoL was considered traumatizing and that informing the dying family member of their diagnosis and/or prognosis would hasten their death due to the discomfort and uneasiness that knowing would create [35, 37]. According to Lee et al. [17], because discussing death is considered disrespectful, it is implied that services like ACP are unacceptable and go against the principle of filial piety in the Chinese culture. The younger participants in Lee et al.'s [17] study revealed that the adult children believed they would upset the senior family member if they were to engage in ACP. Likewise, the older participants also expressed the belief that EoL discussion would be unwelcomed by their children and would instead increase the amount of burden on the family [17].

Alternatively, both older Asian Americans and their families often engage in implicit communication and assumptions regarding EoL treatment. In collectivistic societies where group harmony is emphasized, explicit disclosure of one's emotions and thoughts--especially about adverse topics, is avoided to maintain a sense of peace within the relationships. Alternatively, various Asian cultures engage in nonverbal communication. For example, the term zhihyi in Chinese culture implies one to simply know what the other person thinks [37]. Comparatively, the Japanese culture also have a similar saying--ishin denshin--further implying that one should know without being told [37]. Identically, Koreans have the term nunchi, which signifies understanding through more social, nonverbal cues [37]. As a result, the lack of participation in ACP is thought to be in part the result of both the older individual and the family mutually engaging in implicit, nonverbal communication as form of filial respect and obligation to protect the in-group from perceived harm.

\subsubsection{Acculturation}

Furthermore, the present findings suggests that there is a correlation between ACP, utilization of EoL care treatment, and an individual's degree of acculturation. The samples reveal that in multiple Asian American subgroups, the older adult's degree of acculturation impacted their 
attitudes and willingness to engage in such practices [35, 40]. For instance, third generation Japanese Americans who generally spoke English were more open to ACP and discussing the EoL compared to the other Asian American communities who lacked English proficiency [40]. Moreover, when comparing older Korean Americans and older Koreans living in Korea, those living in America were more highly adapted to the Western culture and thus, had more positive attitudes towards ACP and AD [35]. Therefore, it may be implied that the higher the degree of acculturation, the more likely the older adult and/or their family will be open to engaging in ACP and EoL care treatments.

\subsection{Preferences for End-of-life Decision-Making}

\subsubsection{Preferred Decision-Maker}

Previous studies have suggested that older Asian American ethnic groups prefer family members to make EoL decision on their behalf. Older Asian Americans tend to be uncomfortable with discussing death-related matters because of their cultural backgrounds and upbringing. Many of these individuals also engage in filial expectations for children to make decisions for them. The existing research posits that the family, typically the eldest son, is the preferred decision-maker for many older Filipino and Vietnamese Americans [26, 36, 39]. Research on older Korean Americans revealed expectations for their children to know their wishes at the EoL despite not having direct communication [35]. According to Hong, Yi, Johnson, and Adamek [50], the older Korean American participants specifically identified family members as the final decision-maker regarding their EoL treatment. On the other hand, research by Park [35] suggested that some older Koreans may also choose their physicians as their primary decision-maker out of trust and respect because they believe that medical professionals are more knowledgeable and experienced in health care. Family members have also been consistently recognized as the preferred decision-maker at the EoL among older Chinese and Japanese Americans [37]. However, research studies have demonstrated that some Japanese Americans who speak Japanese are more deferential to physicians as well and prefer having a single individual make EoL decisions on their behalf $[37,40,51]$. In some cases, the family's decision may override their dying parents' preferences for treatment at the EoL are even when those choices are explicitly expressed [50].

\subsubsection{Preferred Location of Care and Death}

Generally, the existing research stipulates that older Asian American adults wish to receive EoL care and die in their own homes. However, research indicates that they ultimately pass away in the hospital setting often [30, 50,51]. According to Matsui and Braun [51], a significant number of Japanese Americans were found to prefer EoL care at home. Hong et al. [50] also found similar preferences from their sample of older Japanese Americans where they expressed the desire for relatives to provide them with care at the EoL in their home. Although a majority of older Asian Americans may wish to die at home, they also strongly believe that dying at home would negatively affect their families emotionally, financially, and spiritually [16, 30, 39].

Contrastingly, research studies have demonstrated that older Chinese Americans may outwardly display the desire to pass in the hospital setting to avoid burdening their family [25, 30]. Similarly, older Vietnamese Americans also believe that passing at home would bring about 
misfortune and invite bad spirits to the home, thus negatively impacting their family [39]. Nonetheless, variations exist amongst older Vietnamese Americans where some express adverse feelings about passing in the hospital due to spiritual beliefs that the soul will wander if one does not pass at home [43].

The existing analyses on older Filipino Americans and their preferences for EoL care is minimal. However, research does indicate that older Filipino Americans are more open to utilizing hospice care as many are more Westernized due to Spanish colonization, more likely to speak English, and are predominantly Roman Catholics who believe death is the ultimate union with God [52]. Therefore, it may be implied that older Filipino Americans are more likely to be open to dying at home because they tend to be more open to using hospice care as compared to other Asian Americans.

\subsubsection{Preferred Treatment}

Furthermore, the current research suggests that older Asian American ethnic groups would prefer not to prolong the dying process with unnatural forms of treatment. Matsui and Braun [51] and Bell et al. [53] illustrated in their studies that the Japanese American participants did not wish to extend their dying with aggressive treatment and instead preferred comfort-based care. Research on older Chinese Americans have also demonstrated that these individuals may not prefer to engage in Western pain-management at the EoL for fear of losing the ability to communicate, to avoid family knowing they are in pain, and to lessen the burden on nurses and doctors [16]. Furthermore, according to Enguidanos et al. [30], it was found that upon learning about hospice care, older Chinese adults were supportive and in favour of the services but became less so after learning that it generally takes place in a home setting and involves the family. Moreover, the Chinese participants believed that hospital-like settings would provide the best quality care whereas nursing homes would have the poorest care [30]. As discussed earlier, older Filipino Americans are also more likely to be open to hospice care and forgoing life-prolonging treatment in accordance with their religious beliefs [36, 52]. In addition, Blackhall et al. [49] discovered that although older Korean Americans were found to believe that life-support mechanisms should be continued under all circumstances and situations, they did not personally desire it for themselves. Thus, it may be implied that the preferred treatment of these groups is dependent on their attitudes towards Western medical practices, their understanding of EoL treatments, and their religious and spiritual beliefs.

The current findings also indicate that older adults from Asian American subpopulations are less likely to desire or engage in pain management at the EoL. According to Duke and Petersen [16], their study that examined older Chinese, Japanese, and Vietnamese American patients' engagement in pain management at the EoL revealed several concerns preventing effective treatment of pain in these communities. For instance, the participants expressed concerns about addiction and the possible side effects of Western medication. The older Chinese adults conveyed worries of becoming tolerant to the pain medications and eventually developing an addiction or reliance towards the drugs [16]. Additionally, the older Chinese participants also feared that because Western medicine is more aggressive than traditional herbal medicines, that they may lose their ability to communicate and experience other adverse effects as a result [16]. The older Japanese and Vietnamese Americans also shared this fear that Western medication would be too 
aggressive. However, both Chinese and Japanese older adults agreed that experiencing unbearable pain would be a strong reason to use Western pain medication [16]. Therefore, the actual experience of pain may provoke usage of pain medication despite personal fears of the potential adverse effects in some older Asian American ethnic groups.

\subsection{Barriers to Advance Care Planning and End-of-Life Care}

In addition to cultural influences, various barriers impede older Asian American ethnic groups from learning about and engaging in ACP and other EoL care treatments. Barriers are unique circumstances that may hinder one's access or working progress towards achieving a desired outcome and exists on different domains, including psychological and structural barriers. The present research indicates that a sense of perceived burden on an emotional and financial level, as well as an absence of culturally-appropriate resources and linguistic barriers to communication prevent many older Asian American adults from obtaining the accurate information and knowledge needed to engage in ACP and EoL care treatment. As a result, rates of participation in EoL care planning remain significantly low in these older Asian subpopulations $[26,38]$.

\subsubsection{Perceived Burden}

The fear of becoming a burden to the family is one of the most commonly identified barriers to communicating EoL preferences among older Asian American ethnic groups. In relation to filial piety, talking about death and dying is believed to be disrespectful and unwelcomed in most Asian American cultures, and therefore thought to bring about emotional and financial burden upon the individual and their family. Duke and Petersen [16] found that both older Chinese and Japanese patients nearing the EoL were less willing to communicate their experience of pain because they did not wish to emotionally burden the family. Moreover, if the appearance of pain is obvious to the family, only then were the older Japanese adults willing to utilize pain medication to lessen their family's distress or to not appear weak [16]. Likewise, older Chinese Americans were also worried that passing away in one's home would psychologically traumatize their family with lasting images of the event [30].

Additionally, older Chinese Americans reported concerns over their property value if they were to pass away in their homes--believing that it would make it harder for their family to sell in the future if needed [30]. Existing research on older Chinese Americans' enrollment in hospice care indicates that some older adults view the service as a way to lessen the burden on the government regarding cost and labour, especially since a large majority of these individuals are enrolled in government supported programs including Medicare and Medicaid [30]. Furthermore, older Asian American ethnic groups were discovered to be concerned about being perceived as a burden to nurses, physicians, and other authority figures. As stated by Duke and Petersen [16], older Chinese and Japanese Americans did not want to share their experiences of pain or request for pain relief to be "good patients" who require little attention and maintenance. The older Chinese participants were less likely to disclose their pain because they wanted to avoid becoming an additional burden to nurses and physicians by being "easy to deal with" instead [16]. As a result, the older patients reported feeling a sense of hesitancy when reporting their needs [16]. 


\subsubsection{Lack of Culturally Sensitive Resources}

Research consistently suggests that older Asian American ethnic groups experience health disparities due to a lack of culturally sensitive and translated medical resources, as well as trained bilingual medical interpreters and translators [15]. According to Ye et al. [15], more than 36 percent of Asian Americans are linguistically isolated in their communities. The lack of suitable resources for these older Asian American adults result in health disparate outcomes that can be detrimental to their health. In a 2007 study examining Chinese and Korean elders' health- care related quality of life, limited English ability was associated with poorer health outcomes [14]. Moreover, the absence of culturally and linguistically sensitive material and support makes it difficult for older Asian American minorities to access the EoL care they need or desire [17].

\subsubsection{Language Barriers}

Correspondingly, language barrier is a central deterrent to EoL care access and knowledge for many older Asian American ethnic groups. For instance, Park [35] found that older Korean immigrants reported being more hesitant to complete legal health documents, such as an AD, because they were unable to sufficiently understand the mechanics of the form. Duke and Petersen's [16] sample of older Japanese Americans reported that if language were not a barrier, they would be more likely and open to expressing experiences of pain at the EoL. In a study examining the communication needs of Chinese American breast cancer patients, language barrier was consistently expressed by the older adults as contributing to poor communication with their physician and poor understanding of health care related concepts [54]. Moreover, research shows that Chinese and Vietnamese patients who lacked English proficiency refrained from inquiring about their health condition compared to their counterparts with higher English fluency [55]. However, language barriers were found not to be an inhibiting factor for Filipino and Japanese Americans. The existing research posits that language barrier may be less of an obstacle for Filipino and Japanese Americans who have higher English proficiency compared to other Asian ethnic groups $[18,38]$.

Moreover, older Asian Americans often depend on their children or other family members for access to social and health-related services, such as EoL care, due to their poor English ability [35]. Oftentimes, children act as interpreters for their older Asian American parents during medical examinations and visits [56]. However, children and other family members acting as interpreters for their parents presents several concerns. These concerns include disrupting the traditional family hierarchy, a general sense of embarrassment or shame by the parent and/or children, inaccurate communication when family interpreters lack the understanding of medical terminology, and a delay in care for the older adult who do not wish to burden their busy children $[17,31]$. Wen et al. [55], also suggested that children as interpreters may not be the best solution due to them not being medically trained and thus, likely to omit important information in the best interest of the parent or because of the lack understanding of medical terminology. This in turn affects the older adults' accurate understanding of their diagnosis, prognosis, and what treatment is available to them at the EoL. As noted earlier, it is suggested that family acting as interpreters for the older adult at the EoL may be particularly common in older Asian American ethnic groups 
who practice filial obligation and have preference for family as surrogate decision makers due to cultural expectations and limited English ability.

Furthermore, research indicates that low health literacy in these older ethnic populations may present an additional barrier to accessing and understanding healthcare and social services like ACP. In the 2007 California Health Interview Study, the degree of health literacy in different Asian American subpopulations were analysed. The results indicated that Chinese Americans with limited English proficiency had the highest prevalence of low health literacy $(n=304,68.3 \%)$ followed by Korean Americans ( $n=329,35.6 \%$ ), and Vietnamese Americans ( $n=215,29.6 \%$ ) [57]. Similarly, according to Tsoh et al. [58], 71\% of 705 surveyed older Chinese American immigrants expressed a need for assistance when reading written health information, despite their primary care physician being Chinese and the primary spoken language between them being Chinese, as well.

Table 1 Summary of research review results: Cultural patterns of EoL care and planning among Asian American ethnic groups

\begin{tabular}{|c|c|c|c|}
\hline & Theme & $\begin{array}{l}\text { Subpopulations } \\
\text { (in alphabetical order) }\end{array}$ & $\begin{array}{l}\text { Sources (in } \\
\text { chronological } \\
\text { order) }\end{array}$ \\
\hline \multirow[t]{2}{*}{$\begin{array}{l}\text { Attitudes towards ACP } \\
\text { and EoL discussions }\end{array}$} & Positive & $\begin{array}{l}\text { Chinese, Filipino, Japanese } \\
\text { Americans }\end{array}$ & $\begin{array}{l}{[17,36,37,} \\
40]\end{array}$ \\
\hline & Negative & Chinese, Vietnamese American & {$[37,39]$} \\
\hline \multirow[t]{5}{*}{ Preferences } & $\begin{array}{l}\text { Preferred decision-maker: } \\
\text { family }\end{array}$ & $\begin{array}{l}\text { Chinese, Filipino, Japanese, Korean, } \\
\text { Vietnamese Americans }\end{array}$ & $\begin{array}{l}{[26,36,37,} \\
39,50]\end{array}$ \\
\hline & $\begin{array}{l}\text { Preference for physicians as } \\
\text { decision-makers }\end{array}$ & Japanese, Korean American & {$[35,37,51]$} \\
\hline & $\begin{array}{l}\text { Preferred location to receive } \\
\text { EoL care and to die: home }\end{array}$ & Chinese, Filipino, Japanese & $\begin{array}{l}{[25,30,41,} \\
50]\end{array}$ \\
\hline & $\begin{array}{l}\text { Preference for foregoing } \\
\text { aggressive life-prolonging } \\
\text { treatment }\end{array}$ & Filipino, Japanese, Korean Americans & {$[36,45,51-53]$} \\
\hline & $\begin{array}{l}\text { Preference to avoid Western } \\
\text { pain-management and } \\
\text { medication }\end{array}$ & Chinese, Japanese American & [16] \\
\hline \multirow[t]{4}{*}{ Cultural Influences } & $\begin{array}{l}\text { Death and illness perceived } \\
\text { as: misfortune or taboo }\end{array}$ & Chinese, Filipino, Korean American & {$[35,37,41]$} \\
\hline & $\begin{array}{l}\text { Death and illness perceived } \\
\text { as: a natural even in life, } \\
\text { inevitable and a will of God } \\
\text { or a higher figure }\end{array}$ & $\begin{array}{l}\text { Filipino, Japanese, Vietnamese } \\
\text { American }\end{array}$ & {$[36,39,42]$} \\
\hline & $\begin{array}{l}\text { Cultural expectation to } \\
\text { endure pain }\end{array}$ & Filipino, Vietnamese American & {$[16,36]$} \\
\hline & Filial piety & $\begin{array}{l}\text { Chinese, Filipino, Japanese, Korean, } \\
\text { Vietnamese American }\end{array}$ & $\begin{array}{l}{[17,36,42,} \\
44,49]\end{array}$ \\
\hline \multirow[t]{2}{*}{ Barriers } & $\begin{array}{l}\text { Emotional and financial } \\
\text { burden }\end{array}$ & Chinese, Japanese & {$[16,30]$} \\
\hline & $\begin{array}{l}\text { Language barrier and health } \\
\text { literacy }\end{array}$ & $\begin{array}{l}\text { Chinese, Japanese, Korean, } \\
\text { Vietnamese }\end{array}$ & $\begin{array}{l}{[15-17,31,38,} \\
44,55,57]\end{array}$ \\
\hline
\end{tabular}




\section{Discussion}

The present findings posit that although a number of older Asian Americans are open to the idea of ACP and EoL care, members of these communities may explicitly express otherwise. The fear of becoming a burden to one's family is a recurrent concept in Asian American subgroups. Due to this perceived worry, older Asian American adults are less likely to outwardly convey experiences of pain or discomfort, and even less likely to initiate discussions about EoL care for fear of emotionally and financially burdening family members. Despite that, the reviewed studies indicate that many older Asian Americans expect or wish for their family to make the final decisions for them at the EoL. However, because both the older individual and their family perceived each other as unwilling to and as feeling uncomfortable discussing death and dying, neither are likely to initiate communication until it may be too late or there is a serious change in health condition.

The assumptions between many older adults, especially Asian Americans, and their families pose concerns about misunderstandings because there are no actual conversations occurring. The lack of communication makes it difficult for family members to truly know what their dying loved one desire in their final moments. Not knowing exactly what the older individual wants at the EoL may inflict even more feelings of burden than it would have if both parties clearly expressed their expectations for one another. At the same time, families may instead opt for life-sustaining treatment for their parents, despite their preferences to do otherwise, because they may feel that they are "not living up to their obligations for caring for their parents unless they try or continue aggressive treatment" [59]. Without actual communication between the older adult and their family, truly knowing what is desired at the EoL remains uncertain.

Language barrier was identified as a significant challenge that ultimately prevented older Asian American ethnic groups from utilizing ACP and EoL care treatment. Correspondingly, limited English proficiency and absence of linguistically concordant resources to meet the needs of each unique subpopulation has a direct impact on how knowledgeable an individual can be about ACP and EoL care services such as hospice. Misconceptions about treatment develop because of incorrect information or no information at all, further reinforcing the underutilization in EoL care services.

Subsequently, the presented findings suggest that information and resources not only need to be linguistically consistent, but should also be delivered in a comprehensible way for those with lower health literacy. More importantly, health literacy is not an issue that is solely experienced by ethnic minority groups lacking English proficiency, but $36 \%$ of all Americans have been found to have elementary or below-elementary understanding of health literacy [60]. Clear understanding of health and healthcare information is essential to the comprehending the available options during the decision-making process involved in ACP and EoL care. Therefore, both limited English proficiency and lower health literacy are primary contributors to the lack of ACP awareness and participation by these populations that need to be further examined and addressed.

\subsection{Implications for Future Research}

Based on the current review, a need exists for further studies on different older Asian American subpopulations. Specifically, there is a dire need for more understanding about older Filipino and 
Vietnamese Americans and their preferences for care and treatment at the EoL. Of all the collected data, specific research about these two subgroups were the most challenging to obtain. Furthermore, different racial and ethnic groups have distinct preferences in participating in research studies. Therefore, further knowledge about culturally appropriate techniques for outreach to these communities is necessary to increase quality understanding of these older Asian American ethnic groups and the EoL. Additionally, future research on ACP and EoL care engagement should produce survey materials in varying languages to achieve increased participation from members of diverse older Asian American communities. These studies should utilize a combination of quantitative and qualitative methods in order to gain a clearer understanding of the attitudes and preferences of these subgroups. Additionally, more qualitative data collection would better enable researchers to determine the extent of older Asian American ethnic groups' understanding of what ACP and EoL care entails, as well as the unique individual variations.

Secondly, due to the lack of initiative by both the older individual and their family member, it may be up to physicians, other health care professionals and even social service agencies to provide the proper educational opportunities that will facilitate EoL care planning. This strategy may be particularly effective because many older adults will wait until there is a serious change in their health status before seeking help and support. Therefore, physicians should carefully monitor patients who may exhibit signs of serious illness or distinct health changes to initiate EoL care discussions in a timely manner that allows both the individual and their family time to think over their options before making any informed decisions. However, research suggests that physicians themselves are also uncomfortable with discussing the EoL due to personal discomfort, being traditionally trained to cure, rather than care academically, and a lack of preparedness for dealing with these situations because of an absence of academic training in such fields of care [61, 62]. Thus, physicians, health care professionals, and public social workers require proper education and training to deliver quality discussion and care to the older individual and their family.

Lastly, a need to investigate the role of acculturation is necessary to understand how the length of time and adaptation to Western norms affects older Asian American adult's attitudes and preferences towards ACP and EoL care. Some research suggests that there is a gradual shift in EoL communication preferences as result of acculturation [63]. For instance, some older Asian immigrants who arrived in the United States at an early age may have significantly adapted to the Western lifestyle and have adjusted their traditional beliefs and practices to better accommodate to their new environment. Thus, while they may maintain their traditional Asian culture, the extent to which they practice them is dependent on their level of acculturation. More specifically, extensive research should be conducted on the role of acculturation in older Vietnamese immigrants who came to the U.S. as refugees and may differ in their attitudes and preferences compared to others. Therefore, it is crucial for all future studies examining older Asian Americans' attitudes on the Eol to gauge the participants' acculturation level because variations exist even between members of the same subpopulation. 


\section{Conclusions}

In this review of diverse older Asian American subpopulations' attitudes and participation in $\mathrm{ACP}$ and EoL care, various commonalities and distinctions were identified amongst these ethnic communities. Although these subgroups may share similar cultural attitudes, barriers, and preferences towards ACP and the EoL, the findings in this study proves that variations not only exist between each general subpopulation, but also within. Therefore, an individual's response to Eol care discussions cannot be assumed by general attitudes and cultural stereotypes. Instead, person-centered approaches to care and communication must be employed in order to achieve higher quality care in the lives of all individuals in any stages of health.

\section{Author Contributions}

All participating authors contributed equally to this work.

\section{Competing Interests}

The authors have declared that no competing interests exist.

\section{References}

1. Sudore R, Lum H, You J, Hanson L, Meier D, Pantilat S, et al. Defining advance care planning for adults: A consensus definition from a multidisciplinary delphi panel. J Pain Symptom Manage. 2017; 53: 821-832.e1.

2. Vincent G, Velkoff V. The next four decades: The older population in the United States: 2010 to 2050 (Current population reports. Series P-25, Population estimates and projections; no. 1138). Washington, D.C.]: [U.S. Dept. of Commerce, Economics and Statistics Administration, U.S. Census Bureau]; 2010.

3. Carr D, Luth E. Advance care planning: Contemporary issues and future directions. Innov Aging. 2017; 1.

4. Heron M. Deaths: Leading causes for 2015. Natl Vital Stat Rep. 2017; 66: 1-76

5. Moody H, Sasser J. Aging: Concepts and controversies (8th ed.). Los Angeles, CA: SAGE; 2015.

6. Detering $K$, Hancock $A$, Reade $M$, Silvester $W$. The impact of advance care planning on end of life care in elderly patients: Randomized controlled trial. BMJ-Brit Med J. 2010; 340: 1-9.

7. Olick R. Defining features of advance directives in law and clinical practice. (Medical Ethics). Chest. 2012; 141: 232-238.

8. Rao J, Anderson L, Lin F, Laux J. Completion of advance directives among U.S. consumers. Am J Prev Med. 2014; 46: 65-70.

9. Bridges J, Lynch T, Schuster A, Crossnohere N, Smith K, Aslakson R. A review of paper-based advance care planning aids. Bmc Palliat Care. 2018; 17: 1-9.

10. Zwakman M, Jabbarian LJ, van Delden JJM, van der Heide A, Korfage IJ, Pollock K, et al. Advance care planning: A systematic review about experiences of patients with a lifethreatening or life-limiting illness. Palliative Med. 2018; 32: 1305-1321. 
11. Where do Americans die? Palliative Care: Education \& Training. 2013. Retrieved April 4, 2018, from https://palliative.stanford.edu/home-hospice-home-care-of-the-dying-patient/wheredo-americans-die/

12. Hamel L, Wu B, Brodie M. Views and experiences with end-of-life medical care in Japan, Italy, the United States, and Brazil: A cross-country survey. 2017. Retrieved from http://files.kff.org/attachment/Report-Views-and-Experiences-with-End-of-Life\%20MedicalCare-in-Japan-Italy-the-United-States-and-Brazil

13. Fonseca L, Testoni I. The emergence of thanatology and current practice in death education. OMEGA - J Death Dying. 2012; 64: 157-169.

14. Mui AC, Kang SY, Kang D, Domanski MD. English language proficiency and health-related quality of life among Chinese and Korean immigrant elders. Health Soc Work. 2007; 32: 119127.

15. Ye J, Mack D, Fry-Johnson Y, Parker K. Health care access and utilization among US-born and foreign-born Asian Americans. J Immigrant Minority Health. 2012; 14: 731-737.

16. Duke G, Petersen S. Perspectives of Asians living in Texas on pain management in the last days of life. Int J Palliat Nurs. 2015; 21: 24-34.

17. Lee $M$, Byon $H$, Hinderer $K$, Alexander $C$. Beliefs in advance care planning among Chinese Americans: Similarities and differences between the younger and older generations. Asian/Pacific Island Nurs J. 2017; 2: 83-90.

18. López G, Ruiz N, Patten E. Key facts about Asian Americans, a diverse and growing population. Pew Research Center. 2017. Retrieved April 4, 2018, from http://www.pewresearch.org/facttank/2017/09/08/key-facts-about-asian-americans/

19. U.S. Census Bureau. Quick Facts. 2017. [online] Available at: https://www.census.gov/quickfacts/fact/table/US/PST045217\#qf-headnote-a [Accessed 1 Mar. 2019].

20. Pew Research Center. Asian Americans. 2019. [online] Available at: http://www.pewresearch.org/topics/asian-americans/ [Accessed 6 Mar. 2019].

21. Acciai $F$, Noah A, Firebaugh G. Pinpointing the sources of the Asian mortality advantage in the USA. J Epidemiol Commun Health. 2015; 69: 1006-1011.

22. Yoo GJ, Musselman E, Lee YS, Yee-Melichar D. Addressing health disparities among older Asian Americans: Data and diversity. Generations. 2015; 8: 74-81.

23. Ngo-Metzger Q, Mccarthy E, Burns R, Davis R, Li F, Phillips R. Older Asian Americans and pacific islanders dying of cancer use hospice less frequently than older white patients. Am J Med. 2003; 115: 47-53.

24. America's Health Insurance Plans. Medicare Advantage Demographics Report, 2015. 2018. [online] Available at:

https://www.ahip.org/wp-content/uploads/2018/06/MADemographics_IssueBrief.pdf [Accessed 1 Mar. 2019].

25. National Hospice and Palliative Care Organization. NHPCO Facts and Figures: Hospice Care in America. 2017. Retrieved from

https://www.nhpco.org/sites/default/files/public/Statistics Research/2016 Facts Figures.pdf

26. Kataoka-Yahiro M, Conde F, Wong R. Advance care planning among Asian Americans and Native Hawaiians receiving hemodialysis. Int J Palliat Nurs. 2010; 16: 32-40. 
27. Bennett M. Nursing care at the end of life: 25 years after the passage of the patient selfdetermination act. J Hosp Palliat Nurs. 2016; 18: 550-555.

28. Idziak J. Ethical dilemmas in long-term care (Study Ed.) (2nd Ed). Dubuque, IA: Simon \& Kolz Publishing; 2002.

29. Hospice Action Network. The Medicare Hospice Benefit. National Hospice and Palliative Care Organization. 2015. Retrieved from https://www.nhpco.org/sites/default/files/public/communications/Outreach/The_Medicare_ Hospice_Benefit.pdf

30. Enguidanos S, Yonashiro-Cho J, Cote S. Knowledge and perceptions of hospice care of Chinese older adults. J Am Geriatr Soc. 2013; 61: 993-998.

31. Aroian K, Wu B, Tran T. Health care and social service use among Chinese Immigrant elders. Res Nurs Health. 2005; 28: 95-105.

32. Jang $Y$, Park N, Chiriboga $D$, Radhakrishnan K, Kim M. The knowing-doing gap in advance directives in Asian Americans: The role of education and acculturation. Am J Hosp Palliat Med. 2017; 34: 874-879.

33. Ko E, Lee J. Completion of advance directives among Korean American and non-Hispanic white older adults. Res Aging. 2010; 32: 618-644.

34. Murphy S, Palmer J, Azen S, Frank G, Michel V, Blackhall L. Ethnicity and advance care directives. J Law Med Ethics. 1996; 24: 108-117.

35. Park J. Advance care planning: A comparison of Korean and Korean American focus groups (California State University, Long Beach. Master's thesis collection, School of Nursing). Long Beach, California]: California State University, Long Beach; 2011.

36. Mcadam J, Stotts N, Padilla G, Puntillo K. Attitudes of critically ill Filipino patients and their families toward advance directives. Am J Crit Care. 2005; 14: 17-25.

37. Chew PK. A case of conflict of cultures: End of-life decision making among Asian Americans. Cardozo J Conflict Res. 2012; 13: 379-391.

38. Jang Y. Asian Americans in Austin: Final report of the Asian American quality of life (AAQoL) survey. 2016. Retrieved from

https://austintexas.gov/sites/default/files/files/Communications/4.2_FINAL_AA_in_Austin_re port_from_UT.pdf

39. Tran C, Hinton L. Health and health care of Vietnamese American older adults. 2010. Retrieved from

http://geriatrics.stanford.edu/wpcontent/uploads/downloads/ethnomed/vietnamese/downloads/vietnamese_american.pdf

40. Matsumura S, Bito S, Liu H, Kahn K, Fukuhara S, Kagawa-Singer M, et al. (2002). Acculturation of attitudes toward end-of-life care - A cross-cultural survey of Japanese Americans and Japanese. J Gen Intern Med. 2002; 17: 531-539.

41. Cruz M, Periyakoil V. Health and health care of Filipino American older adults. Stanford School of Medicine. 2010. Retrieved from http://geriatrics.stanford.edu/ethnomed/filipino

42. Colclough $\mathrm{Y}$, Young $\mathrm{H}$. Decision making at end of life among Japanese American families. J Fam Nurs. 2007; 13: 201-225.

43. American Geriatrics Society. Ethnogeriatrics Committee. Doorway thoughts: Cross-cultural health care for older adults. Sudbury, Mass.: Jones and Bartlett; 2004. 
44. Nguyen G. Generational conflicts among Vietnamese Americans in the health care decision making process. Online J Health Ethics. 2015; 11: 1-15.

45. Shin D, Lee J, Cho B, Yoo S, Kim S, Yoo J. End-of-life communication in Korean older adults: With focus on advance care planning and advance directives. Geriatr Gerontol Int. 2016; 16: 407-415.

46. Fugita S, O'Brien D. Japanese American ethnicity: The persistence of community. Seattle, WA: University of Washington Press; 1991.

47. Shapiro M. Asian culture brief: Philippines. [online] National Technical Assistance Center-Asian Americans and Pacific Islanders. 2003.

Available at: http://www.ntac.hawaii.edu/downloads/products/briefs/culture/pdf/ACB-Vol2Iss3-Philippines.pdf [Accessed 6 Mar. 2019].

48. Lee MC, Hinderer KA, Kehl K. A systematic review of advance directives and advance care planning in Chinese people from eastern and western cultures. J Hosp Palliat Nurs. 2014; 16: 75-85.

49. Blackhall L, Frank G, Murphy S, Michel V, Palmer J, Azen S. Ethnicity and attitudes towards life sustaining technology. Soc Sci Med. 1999; 48: 1779-1789.

50. Hong $M, Y i E$, Johnson KJ, Adamek ME. Facilitators and barriers for advance care planning among ethnic and racial minorities in the U.S.: A systematic review of the current literature. J Immigrant Minority Health. 2018; 20: 1277-1287.

51. Matsui ML, Braun K. Japanese Americans' death attitudes and preferences for end-of-life care. J Hosp Palliat Nurs. 2009; 11: 353-361.

52. Ngo-Metzger Q, Phillips R, McCarthy E. Ethnic disparities in hospice use among AsianAmerican and pacific islander patients dying with cancer. J Am Geriatr Soc. 2008; 56: 139-144.

53. Bell C, Tamura B, Inaba M, Petrovitch H, Ross G, Masaki K. Advance care planning among Japanese-American men aged 89-108 years: The Honolulu-Asian aging study. Gerontologist. 2012; 52: 725-726.

54. Wen K, Hu A, Ma G, Fang C, Daly M. Information and communication needs of Chinese American breast cancer patients: Perspectives on survivorship care planning. J Commun Support Oncol. 2014; 12: 439-445.

55. Green A, Ngo-Metzger Q, Legedza A, Massagli M, Phillips R, lezzoni L. Interpreter services, language concordance, and health care quality. J Gen Intern Med. 2005; 20: 1050-1056.

56. Kim W, Keefe R. Barriers to healthcare among Asian Americans. Soc Work Public Health. 2010; 25: 286-295.

57. Sentell T, Braun K. Low health literacy, limited English proficiency, and health status in Asians, Latinos, and other racial/ethnic groups in California. J Health Commun. 2012; 17: 82-99.

58. Tsoh J, Sentell Y, Gildengorin T, Le G, Chan G, Fung M, et al. Healthcare communication barriers and self-rated health in older Chinese American immigrants. J Commun Health. 2016; 41: 741-752.

59. McCormick AJ. Self-determination, the right to die, and culture: A literature review. Soc Work. 2011; 56: 119-128.

60. Periyakoil V, Neri E, Kraemer H. Patient-reported barriers to high-quality, end-of-life care: a multiethnic, multilingual, mixed-methods study. J Palliat Med. 2016; 19: 373-379.

61. Sullivan AM, Lakoma MD, Block SD. The status of medical education in end-of-life care: $A$ national report. J Gen Intern Med. 2003; 18: 685-695. 
62. Granek L, Krzyzanowska MK, Tozer R, Mazzotta P. Oncologists' strategies and barriers to effective communication about the end of life. J Oncol Pract. 2013; 9: e129-e135.

63. Ahalt C, Walter L, Yourman C, Eng L, Pérez-Stable C, Smith E. "Knowing is better": Preferences of diverse older adults for discussing prognosis. J Gen Intern Med. 2012; 27: 568-575.

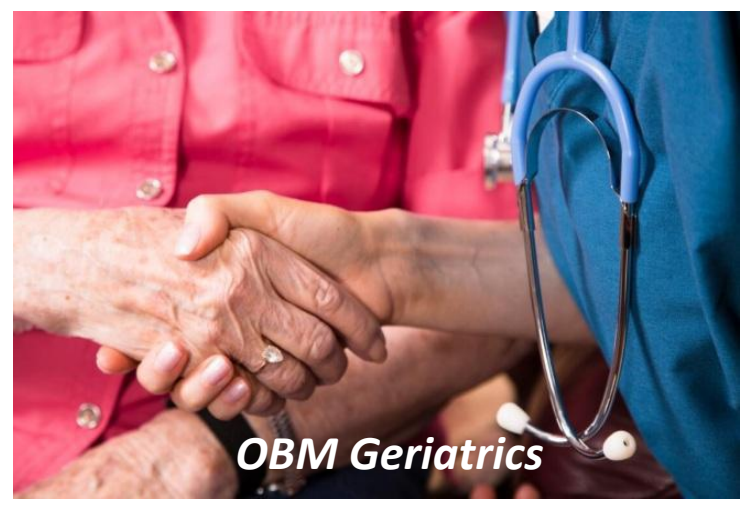

Enjoy OBM Geriatrics by:

1. Submitting a manuscript

2. Joining in volunteer reviewer bank

3. Joining Editorial Board

4. Guest editing a special issue

For more details, please visit:

http://www.lidsen.com/journals/geriatrics 